Julia Lima Fleck

Modelo de Redes Neurais Artificiais para Inferência da Qualidade de um Processo Polimérico

Dissertação apresentada como requisito parcial para obtenção do título de Mestre pelo Programa de PósGraduação em Engenharia Mecânica da PUC-Rio.

Orientadores: Prof. Marcelo de Andrade Dreux

Profa. Belkis Valdman 
Julia Lima Fleck

\section{Modelo de Redes Neurais Artificiais para Inferência da Qualidade de um Processo Polimérico}

Dissertação apresentada como requisito parcial para obtenção do título de Mestre pelo Programa de PósGraduação em Engenharia Mecânica da PUC-Rio. Aprovada pela Comissão Examinadora abaixo assinada.

Prof. Marcelo de Andrade Dreux

Orientador

Departamento de Engenharia Mecânica - PUC-Rio

Profa. Belkis Valdman

Co-orientadora

Departamento de Engenharia Química - EQ/UFRJ

Prof. Maurício Bezerra de Souza, Jr. Departamento de Engenharia Química - EQ/UFRJ

Profa. Marley M. B. Rebuzzi Vellasco Departamento de Engenharia Elétrica - PUC-Rio

Prof. José Eugênio Leal

Coordenador Setorial do Centro Técnico Científico - PUC-Rio

Rio de Janeiro, 22 de agosto de 2008 
Todos os direitos reservados. É proibida a reprodução total ou parcial do trabalho sem autorização da universidade, da autora e dos orientadores.

\section{Julia Lima Fleck}

Graduou-se em Engenharia Química pela Escola de Química da UFRJ. Durante o mestrado, foi bolsista CNPq e pesquisadora no Laboratório de Computação Gráfica Tecgraf em projeto financiado pela Petrobrás.

Ficha Catalográfica

Fleck, Julia Lima

Modelo de Redes Neurais Artificiais para Inferência da Qualidade de um Processo Polimérico / Julia Lima Fleck; orientadores: Marcelo de Andrade Dreux, Belkis Valdman. - 2008.

75f. : il.(colo.); $30 \mathrm{~cm}$

Dissertação (Mestrado em Engenharia Mecânica) Pontifícia Universidade Católica do Rio de Janeiro, Rio de Janeiro, 2008.

Inclui bibliografia.

1. Engenharia Mecânica - Teses. 2. Redes neurais artificiais. 3. Modelagem. 4. Inferência da qualidade. 5. PEBD. I. Dreux, Marcelo de Andrade. II. Valdman, Belkis. III. Pontifícia Universidade Católica do Rio de Janeiro. Departamento de Engenharia Mecânica. III. Título. 


\section{Agradecimentos}

Ao CNPq, pelo auxílio financeiro.

Aos engenheiros Normando de Jesus, Eveline Fraga e Harley Farina, pelo apoio incansável a este trabalho e pelo interesse genuíno em fomentar o desenvolvimento de pesquisas aplicadas à indústria química.

Ao professor José Vitor Bomtempo, da Escola de Química (UFRJ), por todo o auxílio fornecido durante o início deste trabalho.

Aos professores Maurício Bezerra e Marley Vellasco, pelas valiosas sugestões, que foram indispensáveis à melhoria deste trabalho.

Aos meus orientadores, professores Marcelo Dreux e Belkis Valdman, pelo apoio e pela confiança ao longo de todo o desenvolvimento deste trabalho.

Aos meus pais, pelo incentivo constante e inabalável, e por se mostrarem sempre como exemplos de perseverança e competência.

Ao meu noivo, Felipe, pelo carinho e pela cumplicidade ao longo desta e várias outras jornadas. 


\section{Resumo}

Fleck, Julia Lima; Dreux, Marcelo de Andrade. Modelo de Redes Neurais Artificiais para Inferência da Qualidade de um Processo Polimérico. Rio de Janeiro, 2008. 75p. Dissertação de Mestrado - Departamento de Engenharia Mecânica, Pontifícia Universidade Católica do Rio de Janeiro.

O presente trabalho apresenta o desenvolvimento de um modelo neural para a inferência da qualidade do polietileno de baixa densidade (PEBD) a partir dos valores das variáveis de processo do sistema reacional. Para tal, fez-se uso de dados operacionais de uma empresa petroquímica, cujo pré-processamento incluiu a seleção de variáveis, limpeza e normalização dos dados selecionados e preparação dos padrões. A capacidade de inferência do modelo neural desenvolvido neste estudo foi comparada com a de dois modelos fenomenológicos existentes. Para tal, utilizou-se como medida de desempenho o valor do erro médio absoluto percentual dos modelos, tendo como referência valores experimentais do índice de fluidez. Neste contexto, o modelo neural apresentou-se como uma eficiente ferramenta de modelagem da qualidade do sistema reacional de produção do PEBD.

\section{Palavras-chave}

Redes neurais artificiais; Modelagem; Inferência da qualidade; PEBD 


\section{Abstract}

Fleck, Julia Lima; Dreux, Marcelo de Andrade. Artificial Neural Network Modeling for Quality Inference of a Polymerization Process. Rio de Janeiro, 2008. 75p. MSc. Dissertation - Department of Mechanical Engineering, Pontifícia Universidade Católica do Rio de Janeiro.

This work comprises the development of a neural network-based model for quality inference of low density polyethylene (LDPE). Plant data corresponding to the process variables of a petrochemical company's LDPE reactor were used for model development. The data were preprocessed in the following manner: first, the most relevant process variables were selected, then data were conditioned and normalized. The neural network-based model was able to accurately predict the value of the polymer melt index as a function of the process variables. This model's performance was compared with that of two mechanistic models developed from first principles. The comparison was made through the models' mean absolute percentage error, which was calculated with respect to experimental values of the melt index. The results obtained confirm the neural network model's ability to infer values of quality-related measurements of the LDPE reactor.

\section{Keywords}

Artificial neural networks; Modeling; Quality inference; LDPE 


\section{Sumário}

1 Introdução

2 Revisão Bibliográfica $\quad 16$

$\begin{array}{ll}\text { 2.1. Modelos fenomenológicos } & 17\end{array}$

2.2. Modelos empíricos 19

2.3. Modelos baseados no histórico do processo 19

2.4. Redes neurais artificiais 23

2.4.1. Função de ativação 25

2.4.2. Arquitetura 26

2.4.3. Estratégia de aprendizado 26

3 Metodologia $\quad 35$

3.1. Descrição do processo polimérico 35

3.2. Modelagem do processo polimérico 37

3.2.1. Coleta dos dados operacionais 37

3.2.2. Pré-processamento dos dados operacionais 39

3.2.3. Determinação do modelo do processo 44

4 Resultados 49

4.1. Treinamento das redes neurais artificiais 49

4.2. Seleção dos modelos $\quad 50$

4.3. Comparação entre o modelo neural e modelos fenomenológicos 61

$\begin{array}{ll}5 \text { Conclusão } & 68\end{array}$

$\begin{array}{ll}6 \text { Bibliografia } & 70\end{array}$ 


\section{Lista de figuras}

Figura 1 - Classificação dos modelos usados em sistemas

de inferência

Figura 2 - Modelo esquemático de um neurônio artificial (adaptado de Haykin, 1999)

Figura 3 - Representação esquemática do aprendizado com professor (adaptado de Haykin, 1999)

Figura 4 - Representação esquemática de uma rede neural artificial de arquitetura feedfoward (adaptado de Haykin, 1999)

Figura 5 - Representação esquemática da etapa de cálculo forward do treinamento por retropropagação do erro

Figura 6 - Fluxograma simplificado do sistema reacional de produção de PEBD (adaptado de McAuley e MacGregor, 1991)

Figura 7 - Etapas do pré-processamento dos dados operacionais (adaptado de Corrêa, 2005)

Figura 8 - Representação ilustrativa do método hold-out (adaptado de Haykin, 1999)

Figura 9 - Perfil ilustrativo dos erros de treinamento e validação utilizado no treinamento com parada antecipada (adaptado de Haykin, 1999)

Figura 10 - Atividades realizadas durante o desenvolvimento do modelo neural da qualidade do PEBD

Figura 11 - Configuração da rede neural artificial cujo modelo apresentou maior acurácia na modelagem do índice de fluidez do PEBD

Figura 12 - Histograma dos resíduos do índice de fluidez para a rede neural artificial cujo modelo apresentou maior acurácia na modelagem do índice de fluidez do PEBD

Figura 13 - Verificação da constância da variância dos erros (índice de fluidez residual versus índice de fluidez predito pelo modelo neural) 
Figura 14 - Inferência do índice de fluidez do PEBD através do modelo neural proposto neste trabalho

Figura 15 - Inferência do índice de fluidez do PEBD através do modelo fenomenológico utilizado pelo sistema de controle avançado da empresa

Figura 16 - Inferência do índice de fluidez do PEBD através do modelo fenomenológico baseado no modelo de McAuley e MacGregor (1991)

Figura 17 - Análise da correlação entre o valor experimental do índice de fluidez e o valor predito pelo modelo neural proposto no presente trabalho

Figura 18 - Análise da correlação entre o valor experimental do índice de fluidez e o valor predito pelo modelo fenomenológico utilizado pelo sistema de controle avançado Figura 19 - Análise da correlação entre o valor experimental do índice de fluidez e o valor predito pelo modelo fenomenológico adaptado de McAuley e MacGregor (1991) 


\section{Lista de tabelas}

Tabela 1 - Funções de ativação globais 25

Tabela 2 - Função de ativação local 26

Tabela 3 - Seqüência de cálculos realizada a cada apresentação

durante a etapa de cálculo forward do treinamento por

retropropagação do erro

Tabela 4 - Seqüência de cálculos realizada a cada apresentação

durante a etapa de cálculo backward do treinamento por

retropropagação do erro 32

Tabela 5 - Descrição das variáveis selecionadas 40

Tabela 6 - Modelo neural desenvolvido no presente trabalho para inferir o valor do índice de fluidez a partir dos valores das variáveis de processo do sistema reacional de PEBD

Tabela 7 - Erro de validação dos modelos neurais com função de ativação sigmóide na camada escondida (treinamento sem otimização de Levenberg-Marquardt)

Tabela 8 - Erro de validação dos modelos neurais com função de ativação linear na camada escondida (treinamento sem otimização de Levenberg-Marquardt)

Tabela 9 - Erro de validação dos modelos neurais com função de ativação sigmóide na camada escondida (treinamento com otimização de Levenberg-Marquardt)

Tabela 10 - Erro de validação dos modelos neurais com função de ativação linear na camada escondida (treinamento com otimização de Levenberg-Marquardt)

Tabela 11 - Desempenho dos modelos neurais com função de ativação sigmóide na camada escondida (treinamento sem otimização de Levenberg-Marquardt)

Tabela 12 - Desempenho dos modelos neurais com função de ativação linear na camada escondida (treinamento sem otimização de Levenberg-Marquardt) 
Tabela 13 - Desempenho dos modelos neurais com função de ativação sigmóide na camada escondida (treinamento com otimização de Levenberg-Marquardt)

Tabela 14 - Desempenho dos modelos neurais com função de ativação linear na camada escondida (treinamento com otimização de Levenberg-Marquardt)

Tabela 15 - Modelo neural do índice de fluidez do PEBD proposto no presente trabalho

Tabela 16 - Comparação entre a acurácia dos modelos do índice de fluidez do PEBD 